\title{
STELLAR POPULATIONS IN GALAXIES
}

\author{
SIDNEY VAN DEN BERGH \\ David Dunlap Observatory, Toronto, Ontario, Canada
}

\begin{abstract}
There are significant differences among old stellar systems which may partly be a function of luminosity. One such difference is the presence of nuclei in many high luminosity spiral galaxies but their absence in low luminosity irregular systems. Other differences are seen in the spectroscopic and photometric properties.
\end{abstract}

\section{Nuclei of Galaxies}

At first sight the data on old stellar systems suggest that all such objects have very similar morphological, spectroscopic and photometric characteristics. On closer inspection a number of subtle differences become apparent. The major morphological difference between dwarf spheroidal systems on the one hand and giant ellipticals on the other is that giants have nuclei and dwarfs do not. It should, however, be emphasized that not all giant ellipticals have semi-stellar nuclei (King and Minkowski, 1966). The dividing line between galaxies with and without nuclei appears to occur at $M_{V} \simeq-15$. Among the dwarf companions of the Andromeda Nebula NGC 185 $\left(M_{V}=-15.2\right)$ has no nucleus, NGC $147\left(M_{V}=-14.9\right)$ has a faint nucleus and NGC $221=\mathrm{M} 32\left(M_{V}=-16.4\right)$ has a well-developed bright semi-stellar nucleus. The data listed in Table I show that this relation between absolute luminosity and the existence of a nucleus is not confined to elliptical galaxies. The table shows that latetype galaxies of high luminosity are predominantly spirals (which have nuclei) whereas most late-type objects of low luminosity are irregulars which, of course, do not have nuclei.

TABLE I

Relative frequency of spiral and irregular galaxies at different magnitude levels (Van den Bergh, 1966)

\begin{tabular}{lccl}
\hline$\left\langle M_{B}\right\rangle^{\mathrm{a}}$ & Spirals $^{\mathrm{b}}$ & Irregulars & $N(\mathrm{Ir}) / N(\mathrm{Sp})$ \\
\hline-20.2 & 56 & 0 & 0.00 \\
-19.4 & 112 & 1 & 0.01 \\
-18.2 & 66 & 4 & 0.06 \\
$-17.3:$ & 26 & 5 & 0.19 \\
$-15::$ & 22 & 64 & 2.91 \\
\hline
\end{tabular}

a $H=100 \mathrm{~km} \mathrm{~s}^{-1} \mathrm{Mpc}^{-1}$ assumed.

b Barred spirals omitted.

Observations by de Vaucouleurs (1961) and by Tifft (1969) show that the cores of giant $\mathrm{E}$ galaxies are usually redder than their envelopes. McClure's (1969) observations of the intermediate-band cyanogen index $C(41-42)$ show that this colour gradient 
must be due mainly to a change in stellar population rather than to a concentration of dust in the nuclei of E galaxies. McClure's observations of the variation of cyanogen strength within the central regions of M31 and M81 are plotted in Figure 1. These

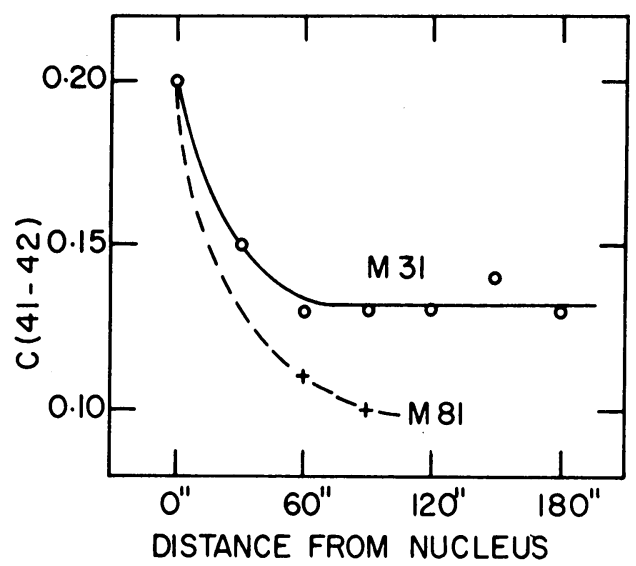

Fig. 1. Variation of cyanogen strength as a function of distance from the nuclei of M31 and M81.

results show that cyanogen is strongly enhanced within a radius of $60^{\prime \prime}(200 \mathrm{pc})$ of the nucleus of M31. Possibly this cyanogen enhancement is in some way related to the steep radial abundance gradient that Peimbert (1968) finds for the nitrogen in the interstellar gas in spiral galaxies. Alternatively it might be assumed that the giant stars in the nuclei of galaxies are somehow related to such nearby cyanogen stars as $\mu$ Leonis and $\alpha$ Serpentis (Spinrad, 1966).

\section{Metal Abundance and Galaxy Luminosity}

Baum (1959) and de Vaucouleurs (1961) have pointed out that the integrated colours of old stellar systems are strongly correlated with their intrinsic luminosities. This effect is illustrated in Figure 2 which shows a plot (McClure and van den Bergh, 1968b) of the reddening-free parameter $Q=\mathrm{U}-\mathrm{B}-0.72(\mathrm{~B}-\mathrm{V})$ vs brightness for $\mathrm{E}$ and $\mathrm{S} 0$ galaxies in the Virgo Cluster. The figure shows that the most luminous $\mathrm{E}$ galaxies are much redder than are fainter ellipticals. The relatively tight correlation between $Q$ and the luminosity of galaxies provides a powerful new tool for the study of the isotropy of the Hubble flow for nearby galaxies.

Available observations (see Table II) show that galaxies of the same absolute magnitude have similar $Q$ values in clusters and in the general field. This result may be interpreted in two different ways: Either (1) the evolutionary history of an E galaxy is independent of the environment in which it is formed i.e. a galaxy is essentially insulated from its neighbours once star formation and heavy element enrichment begin or (2) field E galaxies have escaped from clusters. In connection with the latter 


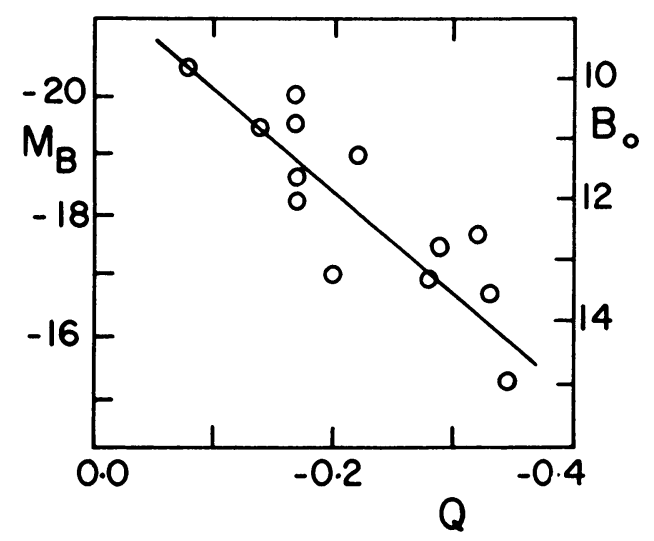

Fig. 2. Plot of the reddening-free parameter $Q$ vs luminosity for $\mathrm{E}$ and $\mathrm{S} 0$ galaxies in the Virgo Cluster.

TABLE II

Colours of cluster and of non-cluster $\mathrm{E}$ galaxies (McClure and Van den Bergh, 1968b)

\begin{tabular}{lll}
\hline Environment & $\langle Q\rangle \mathrm{a}$ & $n_{\text {gal }}$ \\
\hline In rich clusters & $-0.16 \pm 0.01$ & 23 \\
In poor clusters & $-0.18 \pm 0.01$ & 10 \\
Field galaxies & $-0.18 \pm 0.01$ & 27 \\
\hline
\end{tabular}

a All data reduced to $\left\langle M_{B}\right\rangle=-19.6$.

possibility it should be emphasized (Van den Bergh, 1962a) that $76 \%$ of all E galaxies are members of clusters. This observation implies that most clusters containing elliptical galaxies must be stable over time-scales comparable to the lifetime of the Universe.

\section{A. DWARF SPHEROIDAL GALAXIES}

Baum (1959) has pointed out that the integrated colours of dwarf ellipticals and dwarf spheroidal galaxies with $-15<M_{V}<-8$ resemble those of metal-poor halo globular clusters. This observation, in conjunction with available colour-magnitude diagrams for the Draco (Baade and Swope, 1961), Sculptor (Hodge, 1965) and Ursa Minor (Van Agt, 1967) systems makes it virtually certain that the faintest dwarf systems are exceedingly metal-poor. This low metal abundance in dwarf spheroidal systems can probably be understood in terms of the low escape velocity from such objects. Presumably this low escape velocity permitted most of the interstellar matter to escape before a second generation of stars could be formed from enriched interstellar gas.

It has been known for some time (Arp, 1955; Sandage and Wallerstein, 1960) that the red giants in metal-poor galactic globular clusters are brighter than are those 
in metal-rich clusters. A similar correlation between metal abundance and red giant luminosity appears to exist in the globular clusters associated with the Andromeda Nebula (Van den Bergh, 1969). This suggests that a significant spread in the metal abundance of stars in dwarf spheroidal galaxies would manifest itself as a luminosity dispersion in the red giant branch of such a system. Such a dispersion is not seen in Baade and Swope's (1961) observations of the Draco System $\left(M_{V}=-8.6\right)$. Spectroscopic observations (Van den Bergh, 1969) do, however, show evidence for abundance differences among the globular clusters associated with the Fornax dwarf system $\left(M_{V}=-13.6\right)$. This suggests that 'second generation' stars can be formed in systems with masses larger than approximately $1 \times 10^{6} \mathscr{M}_{\odot}$. For elliptical galaxies with masses larger than $10^{9} \mathscr{M}_{\odot}$ the contribution of 'first generation' stars to the total integrated light is swamped by subsequent generations of metal-rich stars.

\section{B. ELLIPTICAL GALAXIES OF INTERMEDIATE LUMINOSITY}

The elliptical galaxy M32=NGC 221, for which $M_{V}=-16.4$, is the best observed early-type galaxy of intermediate luminosity. McClure and Van den Bergh (1968a) find that wide-band UBV photometry and intermediate-band photometry by McClure and by Wood (1966) are well represented by a population model consisting of a dwarfenriched intermediate abundance globular cluster model. Their model, which is compared with observations in Table III, contains the following contributions to V light: globular cluster $47.5 \%$; G8V $25 \%$; K2V $15 \%$; K6V $8 \%$ and M1V $4.5 \%$.

TABLE III

Comparison of the observations of M32 with a dwarfenriched globular cluster model (McClure and Van den Bergh, 1968a)

\begin{tabular}{llr}
\hline Colour index & Observed $^{a}$ & \multicolumn{1}{c}{$O-C$} \\
\hline$C(35-38)$ & & \\
$C(38-41)$ & $-0^{m} .46 \pm 0.03$ & $-0^{m} .01$ \\
$C(41-42)$ & $+0.73 \pm 0.02$ & +0.02 \\
$C(42-45)$ & $+0.08 \pm 0.03$ & 0.00 \\
$C_{35}$ & $+0.78 \pm 0.03$ & +0.01 \\
$C_{41}$ & $+2.21 \pm 0.06$ & 0.00 \\
$C_{47}$ & $+1.09 \pm 0.02$ & -0.01 \\
$C_{55}$ & $+0.47 \pm 0.02$ & +0.02 \\
$C_{60}$ & $+0.04 \pm 0.00$ & 0.00 \\
$C_{67}$ & $-0.16 \pm 0.01$ & -0.01 \\
$C_{73}$ & $-0.34 \pm 0.03$ & 0.00 \\
$L_{52}$ & $-0.54 \pm 0.22$ & +0.09 \\
$L_{59}$ & $+0.07 \pm 0.01$ & -0.02 \\
$L_{62}$ & $-0.10 \pm 0.02$ & -0.04 \\
$L_{66}$ & $+0.06 \pm 0.01$ & -0.02 \\
$L_{71}$ & $-0.05 \pm 0.01$ & -0.01 \\
$U-B$ & $-0.04 \pm 0.05$ & 0.00 \\
$B-V$ & +0.45 & 0.06 \\
& +0.83 & 0.00 \\
\hline
\end{tabular}

a Colours corrected for a reddening of $E_{\mathrm{B}-\mathrm{v}}=0.10$. 
In the model observations of the intermediate-abundance globular cluster NGC 6356 (Morgan Class VI, $Q=-0.24, S p=\mathrm{G} 5$ ) were used to provide the globular cluster contribution to the integrated light of M32. It should be emphasized that a satisfactory fit to the observations cannot be obtained by adding main sequence stars to a metal-poor globular cluster base. Early attempts to synthesize the stellar content of M32 by adding red dwarfs to a metal-poor globular cluster base (Roberts, 1956; Baum, 1959) have previously been criticized by Spinrad (1962) because elliptical galaxies have stronger metal lines and weaker hydrogen lines than do metal-poor globular clusters.

From spectrum scans with the Lick 120-in. telescope Spinrad (1970) has obtained evidence for a small radial composition gradient within M32. Spinrad also finds the nuclear region of this small galaxy to be somewhat redder and stronger lined than its outer regions.

\section{GIANT ELLIPTICAL GALAXIES}

The galaxy M86=NGC 4406 in the Virgo Cluster is a good example of a giant elliptical. For $H=100 \mathrm{~km} \mathrm{~s}^{-1} \mathrm{Mpc}^{-1} \mathrm{M} 86$ has an absolute magnitude $M_{V}=-21.0$. The 'redshift' of M86 is $-292 \mathrm{~km} \mathrm{~s}^{-1}$ so that no $K$ corrections need to be applied to the observed colours. Observations show that M86 is typical of giant ellipticals in all five intermediate-band colours of the DDO system. It has also been observed on the UBV system and on Wood's 12-colour system.

Two population models that fit the observations reasonably well are given in Table IV. The colours obtained from these models are compared with observations in Table V. The only significant discrepancy between the observed and the computed colours occurs for the DDO intermediate-band index $C(41-42)$, which measures the strength of the cyanogen band at $\lambda$ 4216. A similar discrepancy is also found for the nuclear region of M31. The only way to remove this discrepancy in M86 (and in M31) is to replace the normal $K$ giants in the model by 'cyanogen rich' stars similar to $\mu$ Leonis and $\alpha$ Serpentis.

\section{TABLE IV}

Population models for the giant elliptical M86 (McClure and Van den Bergh, 1968a)

\begin{tabular}{|c|c|c|}
\hline \multirow[t]{2}{*}{ Star type } & \multicolumn{2}{|c|}{ Percent contribution to $V$ light } \\
\hline & Model A & Model B \\
\hline F5V & 2 & $\mathbf{0}$ \\
\hline GOV & 10.5 & 10.5 \\
\hline G8V & 13.5 & 13.5 \\
\hline $\mathrm{K} 2 \mathrm{~V}$ & 17 & 17 \\
\hline K6V & 12.5 & 12.5 \\
\hline M1V & 3 & 3 \\
\hline M5V & 3 & 3 \\
\hline G8IV & 3 & 3 \\
\hline KOIII & 33 & 33 \\
\hline K5III & 2.5 & 2.5 \\
\hline metal-poor globular cluster & 0 & 2 \\
\hline
\end{tabular}


The two percent contribution of F5V stars to the total visual light of model A was found to be necessary to obtain a good fit to the continuum colours. Such stars are, however, somewhat earlier in spectral type than the main sequence cut-off expected for a metal-rich stellar population with an age $\sim 1 \times 10^{10} \mathrm{yr}$. In model B the F5V stars have been replaced by a small contribution from a metal-poor globular cluster type

\section{TABLE V}

Comparison of population models of M86 with observations (McClure and Van den Bergh, 1968a)

\begin{tabular}{llcr}
\hline Colour index & Observed colour ${ }^{\mathrm{a}}$ & $\begin{array}{l}\text { Model A } \\
O-C\end{array}$ & $\begin{array}{l}\text { Model B } \\
O-C\end{array}$ \\
\hline$C(35-38)$ & $-0.49 \pm 0.04$ & -0.02 & -0.03 \\
$C(38-41)$ & $+0.80 \pm 0.04$ & -0.01 & -0.01 \\
$C(41-42)$ & $+0.14 \pm 0.02$ & +0.06 & +0.06 \\
$C(42-45)$ & $+0.85 \pm 0.00$ & +0.01 & +0.01 \\
$C_{35}$ & $+2.49 \pm 0.03$ & +0.01 & 0.00 \\
$C_{41}$ & $+1.24 \pm 0.02$ & +0.01 & 0.00 \\
$C_{47}$ & $+0.48 \pm 0.01$ & -0.03 & -0.03 \\
$C_{55}$ & $+0.05 \pm 0.01$ & 0.00 & +0.01 \\
$C_{60}$ & $-0.18 \pm 0.01$ & 0.00 & -0.01 \\
$C_{67}$ & $-0.37 \pm 0.02$ & +0.01 & +0.01 \\
$C_{73}$ & $-0.70 \pm 0.06$ & +0.03 & +0.04 \\
$L_{52}$ & $+0.10 \pm 0.01$ & +0.01 & +0.01 \\
$L_{59}$ & $-0.05 \pm 0.01$ & +0.01 & +0.02 \\
$L_{62}$ & $+0.07 \pm 0.01$ & -0.01 & -0.01 \\
$L_{66}$ & $-0.07 \pm 0.02$ & -0.01 & 0.00 \\
$L_{71}$ & $0.00 \pm 0.06$ & +0.01 & +0.01 \\
$\mathrm{U}-\mathrm{B}$ & +0.54 & 0.00 & -0.02 \\
$\mathrm{~B}-\mathrm{V}$ & +0.88 & -0.05 & -0.07 \\
& & &
\end{tabular}

a Observed colours corrected for $E_{\mathrm{B}-\mathrm{v}}=0.06$.

population. The data in Table $\mathrm{V}$ show that model $\mathrm{B}$, in which the globular cluster population gives a two percent contribution to the total light, gives an acceptable fit to the observations. It should be emphasized that the observed and the computed $C(35-38), C(38-41)$ and $\mathrm{U}-\mathrm{B}$ colours can no longer be brought into agreement if more than two percent of the visual light in M86 is contributed by a metal-poor globular cluster population. In an independent investigation Spinrad (1970) finds that population models with a few late $\mathrm{F}$ stars give a somewhat better fit to his spectrum scans than do models with a small metal-poor globular cluster contribution. Perhaps this indicates that the halo population (including globular clusters) is moderately metal-rich in giant elliptical galaxies. A crucial test of this hypothesis will be provided by photometry of globular clusters associated with giant elliptical galaxies. Such a test is currently being undertaken by Racine and Hanes, who are using 200-in. plates to do photographic photometry of the globular clusters associated with M87. 


\section{The Mass-to-Light Ratio in Elliptical Galaxies}

The mass-luminosity relation for dust-poor stellar systems has been discussed by Poveda (1961). His investigation shows that there is a progressive increase in the mass-to-light ratio from globular clusters through dwarf ellipticals to giant E galaxies. The models for M32 and for M86, that were discussed in the previous section, are consistent with Poveda's results. Both models for M86 give $\mathscr{M} / L_{B} \simeq 13$, which is significantly higher than the value $\mathscr{M} / L_{B} \simeq 5$ that is obtained for our model of M32. It should, however, be emphasized that $\mathscr{M} / L_{B}$ could be increased significantly in all models by adding large numbers of M8V stars which do not make a significant contribution to the total $\mathrm{V}$ luminosity of a galaxy.

\section{Stellar Populations in the Galactic Disk}

Observations of the frequency with which main sequence stars of different metal abundance occur near the Sun (Van den Bergh, 1962b) show that the rate of heavy element enrichment in the Galaxy has decreased much faster than the rate of star formation. This observation suggests that the stellar birthrate function initially contained more massive stars (which rapidly become supernovae) than does the present luminosity function of star formation. Rather similar arguments have also been used by Schmidt (1963) to show that the birthrate of massive stars has decreased faster than has the birthrate of low-mass stars. It is not yet clear how this conclusion is to be reconciled with observations of the stellar luminosity function near the Sun.

Comparison of the mean luminosity function of star clusters (Van den Bergh, 1961)

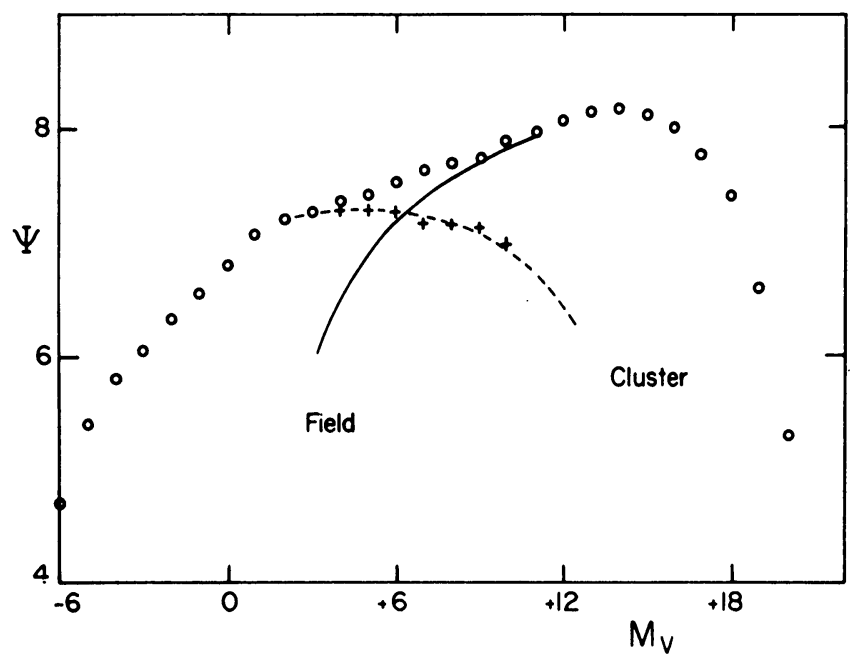

Fig. 3. This figure suggests that the stellar birthrate function near the Sun might consist of two distinct components. 
with the stellar birthrate function $\psi\left(M_{V}\right)$ (Limber, 1960) shows that open clusters contain fewer faint stars than does the luminosity function of field stars near the Sun. This discrepancy appears significant even though rather large differences exist between the luminosity functions of different clusters. This observation shows that the galactic disk cannot have been populated entirely by disintegrating open clusters. In fact Figure 3 suggests that the stars in the galactic disk near the Sun may constitute a mixture of two components: (1) a population derived from the disintegration of open clusters and (2) a 'field star' population. This result might be explained by the ad hoc hypothesis that the luminosity function of star formation in zones of positive energy (associations) contains more faint stars than it does in regions of negative energy (clusters). Alternatively it might be assumed that the 'field stars' represent a very old constituent of the galactic disk population. In the latter case the 'field star' component of the galactic disk might be related to the faint star component that contributes most of the mass in giant ellipticals.

The hypothesis that 'field stars' constitute an old component of the galactic disk leads to an interesting paradox: to explain the observed metal abundance of stars in the galactic disk it is necessary to postulate that the stellar birthrate function initially contained more massive stars than it does now. On the other hand the luminosity function of disk stars suggests that the stellar birthrate function initially contained more low-mass stars than it does now. Perhaps the early history of the Galaxy was much more complex than the situation envisaged in currently fashionable models for galactic evolution.

\section{Differences Among Old Metal-Poor Populations}

Detailed observations of individual stars of Population II are only possible in the Galaxy, in the Magellanic Clouds and in nearby dwarf spheroidal systems. The rather

TABLE VI

Colour-magnitude diagrams of globular clusters in the Magellanic Clouds (Van den Bergh, 1968)

\begin{tabular}{cll} 
Cluster & $\begin{array}{l}\text { Horizontal } \\
\text { branch* }\end{array}$ & $\begin{array}{l}\text { B - V colour at } \\
\text { tip of giant branch }\end{array}$ \\
\hline SMC NGC 121 & $R$ & 1.9 \\
NGC339 & $R$ & 2.2 \\
NGC 361 & - & 2.1 \\
NGC419 & - & 2.7 \\
L1 & $R$ & 2.4 \\
L8 = K3 & $R$ & 2.2 \\
LMC NGC 1466 & $B$ & 1.6 \\
NGC 1783 & $R ?$ & 2.4 \\
NGC 1841 & $B$ & 1.7 \\
NGC 1846 & - & 2.6 \\
NGC 1978 & - & 2.1 \\
NGC 2257 & $B$ & 1.8 \\
\hline$R-$ most of stars to the red of variable star gap, \\
B - most of stars on the blue side of this gap.
\end{tabular}


fragmentary data that are so far available hint at a number of significant differences between galactic and extra-galactic Population II stars. Perhaps the most striking differences that have so far been observed (see Table VI) are those between the globular clusters in the Magellanic Clouds and those in the Galaxy. All the globulars in the Small Cloud and about half of those that have so far been observed in the Large Cloud seem to contain a few exceedingly red giant stars with colour indices in the range $1.9 \leqslant B-V \leqslant 2.7$. Such very red stars are not known to occur in galactic globular clusters. It might be speculated that these very red stars in the Cloud clusters are mild carbon stars. If this is indeed the case they might possibly represent objects in the helium shell burning phase that have managed to mix carbon from their inert carbon-oxygen cores up to their surfaces. In his study of the Sculptor system Hodge (1965) found two very red giant stars that are possibly similar to those that occur in the Small Cloud clusters. This observation suggests that some dwarf spheroidal galaxies may contain a stellar population similar to that which is encountered in the Small Cloud globulars.

An additional argument for differences between Population II stars in the Galaxy and in dwarf spheroidal systems is provided by Van Agt's (1967) observations of BL Herculis stars in dwarf spheroidals. At a given period he finds these variables to be almost a magnitude brighter in dwarf spheroidals than they are in galactic globular clusters.

Tifft's (1963) observations of the 1.43 variable in the Small Cloud cluster NGC 121 gives a distance modulus that is in excellent agreement with that obtained from classical Cepheids if it is placed on Van Agt's period luminosity relation for BL Herculis stars in dwarf spheroidal systems rather than on that for galactic globulars. This observation tends to strengthen the suspicion that the stars in the Cloud globulars are more closely related to those in dwarf spheroidal systems than they are to galactic Population II.

The properties of RR Lyrae variables provide additional evidence for differences between Population II stars in galactic globular clusters and in dwarf spheroidal galaxies. According to Van Agt and Oosterhoff (1959) the mean periods $\langle P\rangle_{a b}$ of RR Lyrae variables in galactic globulars cluster around 0.55 and 0.65 . No such clustering is apparent for dwarf spheroidal systems. In fact two of the four dwarf spheroidals that have so far been studied have $\langle P\rangle_{a b} \simeq 0.60$, which places them halfway between Oosterhoff's long-period and short-period groups.

Dwarf spheroidal galaxies are exceedingly fragile objects. Because of their low space densities such objects can never have approached any of the more massive members of the Local Group very closely. It therefore seems improbable that they could have suffered significant contamination from nucleosynthesis in these massive galaxies. This suggests that individual dwarf spheroidal galaxies probably had a rather simple chemical evolution. It is therefore rather surprising that significant differences appear to exist between the colour-magnitude diagrams of different dwarf spheroidal galaxies. Van Agt's (1967) observations show that the Ursa Minor system has a blue horizontal branch. On the other hand the Draco system (Baade and Swope, 1961) and the Leo II system (Swope, 1967) exhibit red horizontal branches similar to those of the intergalactic globular clusters Pal. 3 and Pal. 4 (Burbidge and Sandage, 1958). 
Available observations of the colour-magnitude diagrams of galactic globular clusters (White, 1970) show that these clusters (with the possible exception of NGC 362) have similarities (family traits?) that suggest a common ancestry. It is, however, quite obvious (Hartwick, 1968) that the galactic globular clusters do not form a one parameter sequence i.e. their 'family tree' has many branches.

It has been suggested (Van den Bergh, 1965, 1967; Sandage and Wildey, 1967) that the parameters which determine the characteristics of globular cluster colourmagnitude diagrams are helium abundance and metal abundance. An alternative suggestion by Rood and Iben (1968) is that the observed differences between globular clusters are due to differences in age and metal abundance. Rood and Iben argue that the age differences among galactic globular clusters might well exceed the $2 \times 10^{8}$ yr collapse time-scale envisioned by Eggen et al. (1962). It turns out, however, that rather extreme assumptions have to be made to obtain age differences as large as $1 \times 10^{9} \mathrm{yr}$. A third possibility, which deserves to be explored in more detail is that the observed differences among Population II objects of similar metal abundance are due to variations in the relative abundances of different species of heavy elements. Such differences might arise if different types of supernovae had differing spatial distributions.

\section{Acknowledgements}

I thank Drs. Robert McClure, Allan Sandage and Hyron Spinrad for a number of stimulating discussions.

\section{References}

Agt, S. L. T. J. van: 1967, Bull. Astron. Inst. Neth. 19, 275.

Agt, S. L. T. J. van and Oosterhoff, P. T.: 1959, Leiden Ann. 21, 253.

Arp, H. C.: 1955, Astron. J. 60, 317.

Baade, W. and Swope, H. H.: 1961, Astron. J. 66, 300.

Baum, W. A.: 1959, Publ. Astron. Soc. Pacific 71, 106.

Bergh, S. van den: 1961, Astrophys. J. 134, 553.

Bergh, S. van den: 1962a, Z. Astrophys. 55, 21.

Bergh, S. van den: 1962b, Astron. J. 67, 486.

Bergh, S. van den: 1965, J. Roy. Astron. Soc. Can. 59, 151.

Bergh, S. van den: 1966, Astron. J. 71, 922.

Bergh, S. van den: 1967, Publ. Astron. Soc. Pacific 79, 460.

Bergh, S. van den: 1968, J. Roy. Astron. Soc. Can. $62,145$.

Bergh, S. van den: 1969, Astrophys. J. Suppl. 19, 145.

Burbidge, E. M. and Sandage, A. R.: 1958, Astrophys. J. 127, 527.

Eggen, O. J., Lynden-Bell, D., and Sandage, A. R.: 1962, Astrophys. J. 136, 748.

Hartwick, F. D. A.: 1968, Astrophys. J. 154, 475.

Hodge, P. W.: 1965, Astrophys. J. 142, 1390.

King, I. R. and Minkowski, R.: 1966, Astrophys. J. 143, 1002.

Limber, D. N.: 1960, Astrophys. J. 131, 168.

McClure, R. D.: 1969, Astron. J. 74, 50.

McClure, R. D. and Bergh, S. van den: 1968a, Astron. J. 73, 313.

McClure, R. D. and Bergh, S. van den: 1968b, Astron. J. 73, 1008.

Peimbert, M.: 1968, Astrophys. J. 154, 33.

Poveda, A.: 1961, Astrophys. J. 134, 910.

Roberts, M. S.: 1956, Astron. J. 61, 195. 
Rood, R. and Iben, I.: 1968, Astrophys. J. 154, 215.

Sandage, A. R. and Wallerstein, G.: 1960, Astrophys. J. 131, 598.

Sandage, A. R. and Wildey, R. L.: 1967, Astrophys. J. 150, 469.

Schmidt, M.: 1963, Astrophys. J. 137, 758.

Spinrad, H.: 1962, Astrophys. J. 135, 715.

Spinrad, H.: 1966, Publ. Astron. Soc. Pacific 78, 367.

Spinrad, H.: 1970, private communication.

Swope, H. H.: 1967, Publ. Astron. Soc. Pacific 79, 439.

Tifft, W. G.: 1963, Monthly Notices Roy. Astron. Soc. 125, 199.

Tifft, W. G.: 1969, Astron. J. 74, 354.

Vaucouleurs, G. de: 1961, Astrophys. J. Suppl. 5, 233.

White, R. E.: 1970, Astrophys. J. Suppl. 19, 343.

Wood, D. B.: 1966, Astrophys. J. 145, 36.

\section{Discussion}

Lewis: Have you taken into account the possible explanation offered by 'collapsars' or 'black holes' to the high $\mathscr{M} / L$ ratios of E's etc.; while these need not contribute to the light they give the high $\mathscr{M} / L$ ratio without resort to the filling of E's with dwarf type stars. The existence of these past super-massive stars would provide a natural explanation of considerable abundances of metals from the past history.

Van den Bergh: No black holes are needed inside galaxies! Stellar population models that fit existing multicolour observations require mass-to-light ratios similar to those that King and Minkowski obtain from dynamic considerations.

King: The King-Minkowski paper will give evidence that there are not enough collapsars to have any appreciable gravitational effect. Regarding the correlation between $Q$ and $M_{B}$, we do not find such a correlation between $\mathscr{M} / L$ and $M_{B}$ for Virgo giants - we see only a difference between giants and dwarfs. We find the $\mathscr{M} / L$ of M86 to be the least extreme in $\mathscr{M} / L$ of all the Virgo giants that we have studied; the others have even higher $\mathscr{M} / L$.

Mrs. Rubin: What is the evidence that the strong $\mathrm{CN}$ or at least some part of it does not arise in interstellar gas in the galaxies? In some galaxies it appears that $\mathrm{Na}$ D-lines may be partly of interstellar origin.

Van den Bergh: I would guess that an unreasonably large amount of interstellar gas would be required to account for the observed cyanogen strength in the nuclei of galaxies.

Tifft: Small aperture four-colour uniform photometry of Virgo ellipticals shows a close colour index correlation with luminosity and supports the $Q, M$ correlation presented by Van den Bergh.

Morgan: In connection with the measures of the CN intensity in giant ellipticals (NGC 4472, etc.) and in the nuclear region of Sb spirals (M31, etc.); have you corrected for the effects of non-CN absorptions of CaI, CrI, etc. from K4-early M giants?

Van den Bergh: Yes, our models (see Table IV) do include K5III stars. Furthermore, recent models of the nucleus of M31 presented by Spinrad at the Rome Symposium contain both K and M giants. These models show the same cyanogen deficiency exhibited by our models.

Mrs. Burbidge: Could you comment on the stellar population in globular clusters in M31? How does the high proportion of metal-rich clusters which you found tie in with the nuclear population?

Van den Bergh: Perhaps the average metal abundance of globular clusters will turn out to depend on the luminosity of their parent galaxy. The globulars in the Fornax dwarf system are all metal-poor. Most of the globular clusters associated with the Galaxy are metal-poor, but there is also a significant number of relatively metal-rich galactic globular clusters. Finally my recent photometric and spectroscopic observations of the globulars in M31 seem to show that these clusters are, on the whole, richer in metals than are those in the Galaxy. It would be interesting to know if the globular clusters associated with such giant E galaxies as M87 are metal-rich. Perhaps the colour observations by Racine and Hanes, to which I referred previously, will provide an answer to this question.

Ekers: Can you place limits on a possible non-stellar light contribution in the giant ellipticals? To what extent could this affect your analysis of stellar content?

Van den Bergh: Available observations now cover a wide wavelength baseline. This places quite severe constraints on the amount of non-thermal background radiation that can be present in normal elliptical galaxies. Galaxies with known peculiarities, such as M87, were avoided during the David Dunlap Observatory observing program. 